\title{
The efficacy of PD-1/PD-L1 inhibitors in advanced squamous-cell lung cancer: a meta-analysis of 3112 patients
}

\author{
Rui-Lian Chen ${ }^{\ddagger 1}$, Jing-Xu Zhou ${ }^{\ddagger}{ }^{1}$, Yang Cao ${ }^{1}$, Sui-Hui Li ${ }^{1}$, Yong-Hao Li ${ }^{1}$, Mei Jiang ${ }^{1}$, \\ Dong-Yan Lu${ }^{1}$, Peng Li ${ }^{1}$ \& Li-Zhu Lin*,1 \\ ${ }^{1}$ Integrative Cancer Centre, the First Affiliated Hospital of Guangzhou University of Chinese Medicine, Guangzhou, China \\ *Author for correspondence: lizhulin26@yahoo.com \\ $\ddagger$ Authors contributed equally
}

\begin{abstract}
Aim: We performed a meta-analysis to explore the efficacy of immunotherapy for patients with squamous non-small-cell lung cancer (NSCLC). Materials \& methods: Randomized clinical trials comparing immunotherapy with chemotherapy for advanced NSCLC patients were included. Results: A total of 11 trials (3112 patients) were included. PD-1/PD-L1 inhibitors demonstrated significant superiority to chemotherapy in overall survival (OS) (hazard ratio [HR]: 0.74; $p<0.001$ ) and progression-free survival (PFS) (HR: 0.66; $p<0.001$ ) for squamous NSCLC. The OS and PFS benefits of PD-1/PD-L1 inhibitors for squamous NSCLC were similar in subgroup analyses of line settings, PD-L1 expression and different study methodologies. No advantage in OS was found in advanced squamous NSCLC patients treated with atezolizumab (HR: 0.87; $p=0.087)$. Conclusion: PD-1/PD-L1 inhibitors significantly improved OS and PFS in advanced squamous NSCLC patients when compared with chemotherapy.
\end{abstract}

First draft submitted: 20 June 2019; Accepted for publication: 14 October 2019; Published online: 12 November 2019

Keywords: chemotherapy $\bullet$ efficacy $\bullet$ lung cancer $\bullet$ meta-analysis $\bullet$ PD-1/PD-L1 inhibitors $\bullet$ squamous

Lung cancer presents the leading cancer type and the major cause of cancer-related mortality worldwide [1]. Adenocarcinoma and squamous carcinoma are two common histological subtypes of non-small-cell lung cancer (NSCLC). Squamous-cell carcinoma accounts for approximately $20-30 \%$ of all cases of lung cancer [2].

The emergency of targeted agents has significantly improved the overall survival (OS) and quality of life for advanced NSCLC patients [3,4]. However, this advance is limited to patients with specific driver gene alterations that are rarely found in squamous-cell NSCLC patients [5]. Most new agents, including bevacizumab and pemetrexed, have been approved for NSCLC but not for the squamous-cell subtype because of their severe toxicities [6,7]. The combination of the EGFR inhibitor necitumumab and chemotherapy as the first-line setting has shown superior OS compared with chemotherapy alone for squamous NSCLC [8]. However, the modest benefit from combination treatment has been associated with added toxicity for squamous-cell NSCLC patients. Thus, limited treatment strategies are available for these patients. Platinum-based doublet chemotherapy has been considered as the standard therapy for metastatic squamous NSCLC, such as nab-paclitaxel plus carboplatin; however, they have only resulted in poor efficacy with a median progression-free survival (PFS) of 6.3 months and a median OS of 12.1 months [9]. Therefore, there is an urgent need to identify more effective prognostic therapeutic regimens for advanced squamous NSCLC patients.

The current development of immune checkpoint inhibitors (ICIs) has become a paradigm shift in the therapeutic strategies of NSCLC patients [10]. The programmed cell death ligand 1 (PD-L1) and programmed cell death 1 (PD-1) pathways present critical immunosuppressive mechanisms. Binding of PD-L1 expressed on tumor cells to PD-1 expressed on T-cells suppresses cytotoxic activity against cancer and leads to tumor immune evasion, dissemination, relapse and metastasis. ICIs treatment can block the interaction between PD-1 and PD-L1, and then the T-cell immune response against cancer cells can be restored [11]. Several trials have shown that patients benefited from antibodies blocking the PD-1/PD-L1 pathway in the management of various cancers [12-14].

Future Medicine 
In a series of clinical trials, PD-1 inhibitors (nivolumab, pembrolizumab and avelumab) and a PD-L1 inhibitor (atezolizumab) are common ICIs that have demonstrated superiority in durable antitumor responses and improved OS compared with chemotherapy for metastatic NSCLC patients [15-18]. A Phase III trial (Checkmate 017) demonstrated that nivolumab significantly prolonged OS for previously treated squamous NSCLC patients when compared with chemotherapy (hazard ratio [HR]: 0.59; 95\% CI: 0.44-0.79; p < 0.001) [19]. Another randomized Phase III trial (KEYNOTE-407) indicated that treatment-naive squamous-cell NSCLC patients treated with a combination of pembrolizumab plus chemotherapy experienced superior PFS and OS versus chemotherapy alone (15.9 vs 11.3 months; $\mathrm{p}<0.001$ ) [20]. However, subgroups analyses of Checkmate 026, KEYNOTE-010 and POPLAR trials showed that squamous-cell lung cancer patients received no significant benefit in OS from PD1/PD-L1 inhibitors when compared with chemotherapy alone [18,21,22]. IMpower 131 trial showed no improved OS in advanced squamous NSCLC patients treated with atezolizumab plus carboplatin and nab-paclitaxel versus those treated with chemotherapy [23].

Meta-analysis is generally considered a powerful statistical tool to overcome the limitations of different sample sizes from individual studies to generate the best overall estimation. Thus, it is necessary to perform a meta-analysis to explore the efficacy of immunotherapy for advanced squamous NSCLC patients. The aim of the current study was to conduct a meta-analysis of all eligible published studies to explore the efficacy of PD-1/PD-L1 inhibitors for advanced squamous-cell lung cancer patients.

\section{Methods}

\section{Data source}

We conducted a systematic search of Pubmed, Embase and the Cochrane library to identify all eligible trials regarding NSCLC, from the inception to each database until 1 May 2019 with no start date limit applied. Literature search terms and related variants were used, including pembrolizumab, nivolumab, atezolizumab, avelumab, recurrent, stage IV, metastasis, advanced, lung cancer, non-small-cell lung carcinoma and NSCLC. Meeting abstracts were also searched from the American Society of Clinical Oncology (ASCO), European Society for Medical Oncology, American Association for Cancer Research for Medical Oncology and World Conference on Lung Cancer.

\section{Study selection}

All eligible studies meeting the following criteria were included: randomized clinical Phase II or III trials in patients with advanced NSCLC; random assignment of chemotherapy or immunotherapy (nivolumab, pembrolizumab, atezolizumab or avelumab) alone or in combination; and reporting the HR for OS and/or PFS for squamous-cell lung cancer from overall patients or subgroups. Observational studies, editorials, reviews and commentaries were excluded. Abstracts and studies written in languages other than English or with insufficient data for estimating HR and $95 \%$ CI were also excluded.

Two investigators ( $\mathrm{SH} \mathrm{Li}$ and DY Lu) independently searched the databases and screened studies by their titles and abstracts to choose potentially relevant articles. The full texts of relevant articles were retrieved for eligibility.

\section{Data extraction}

All candidate articles were evaluated and extracted by two authors (RL Chen and JX Zhou) and all disagreements were resolved by consensus of all investigators. Study characteristics, including first author name, clinical trial name, clinical trial year of publication, trial phase, type of therapy, treatment line setting and number of patients were extracted from each eligible study.

Two investigators (Y Cao and M Jiang) assessed the quality of included trials independently according to the five-point Jadad scoring system [24], which was based on the quality of randomization and double-blinding study design, procedure, sample size estimation, blinding method, withdrawals and dropouts.

\section{Subgroup analysis}

We performed a number of subgroup analyses to explore the variables on immunotherapy efficacy for squamouscell lung cancer. The subgroup included lines of therapy (first-line and subsequent lines), types of PD-1/PD-L1 inhibitors (nivolumab, pembrolizumab and atezolizumab), study methodologies (immunotherapy plus chemotherapy vs immunotherapy alone) and PD-L1 expression. Subgroups including more than two studies were considered in our study. 


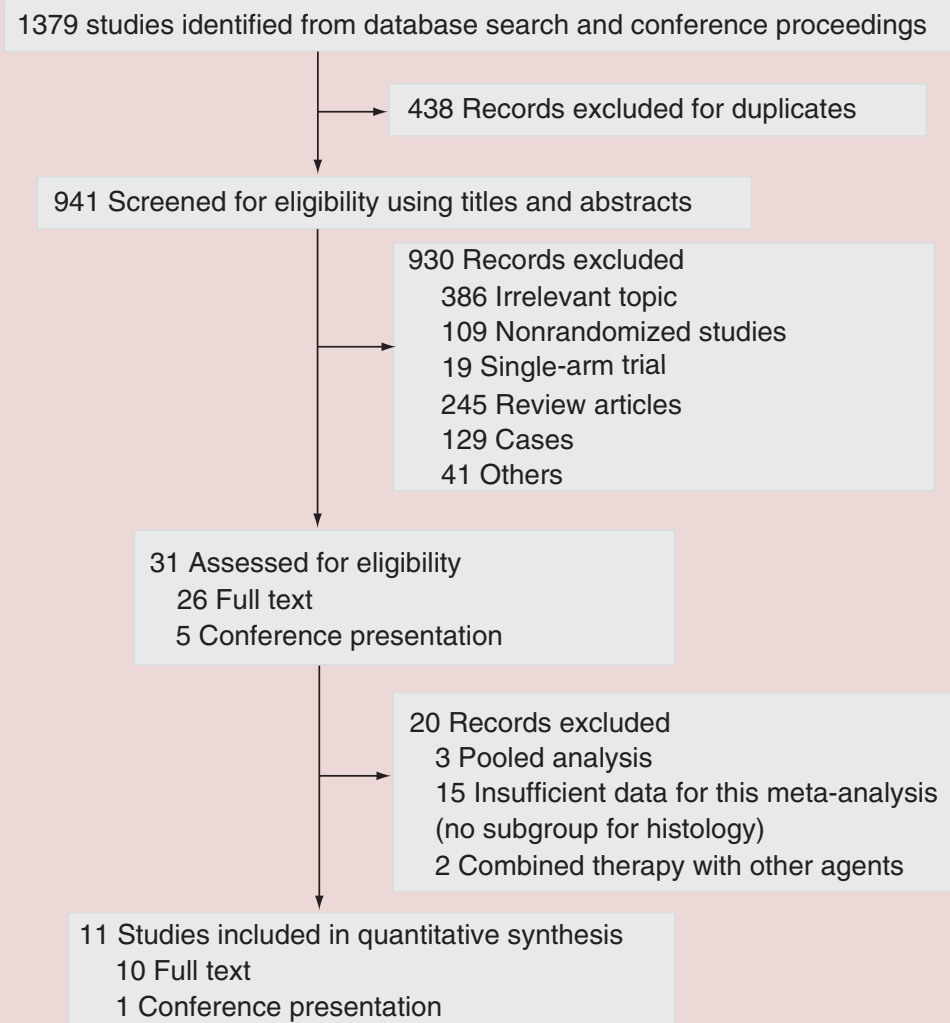

Figure 1. The flowchart of the study selection process for the meta-analysis.

\section{Statistical analysis}

We derived the HRs and 95\% CI for OS and PFS from the overall population and subgroups from each individual trial of advanced squamous-cell lung cancer. Heterogeneity between the studies was assessed using the Q-test and was quantified with $\mathrm{I}^{2}$ values [25]. $\mathrm{I}^{2}$ statistic $>50 \%$ or $\mathrm{p}$-value $<0.05$ was considered indicative of significant heterogeneity between trials. The random-effect model was performed for statistical analysis when significant heterogeneity was observed. The fixed-effect model was used if heterogeneity was not significant. Publication bias was assessed by visual inspection of the funnel plot and the Begg's and Egger's tests. All reported p-values were two sided and $\mathrm{p}<0.05$ was considered as statistically significant. The meta-analysis was performed with Review Manager (RevMan, version 5.3). We followed the recommendations of the Cochrane Collaboration and the Preferred Reporting Items for Systematic Reviews and Meta-Analyses guidelines for this meta-analysis.

\section{Results}

\section{Literature search results}

In total, our search strategy identified 1379 potentially relevant records from databases and conferences. Our selection process and reasons for study exclusion are presented in Figure 1. A total of 1368 studies were excluded after screening the abstract and full text. A total of 11 studies were considered eligible for the current meta-analysis.

\section{Characteristics of the selection studies $[15-23,26,27]$}

A total of 11 clinical trials involving 3112 patients with advanced squamous-cell NSCLC were included in our meta-analysis (1631 in the PD-1/PD-L1 inhibitors group and 1481 in the chemotherapy group). All trials were Phase II or III randomized controlled clinical trials. Among these studies, six were conducted in first-line setting, whereas five were conducted with second or additional lines of therapy. We found seven trials with PD-1 inhibitors (three with nivolumab and four with pembrolizumab) and four with PD-L1 inhibitors (one with avelumab and three with atezolizumab). 


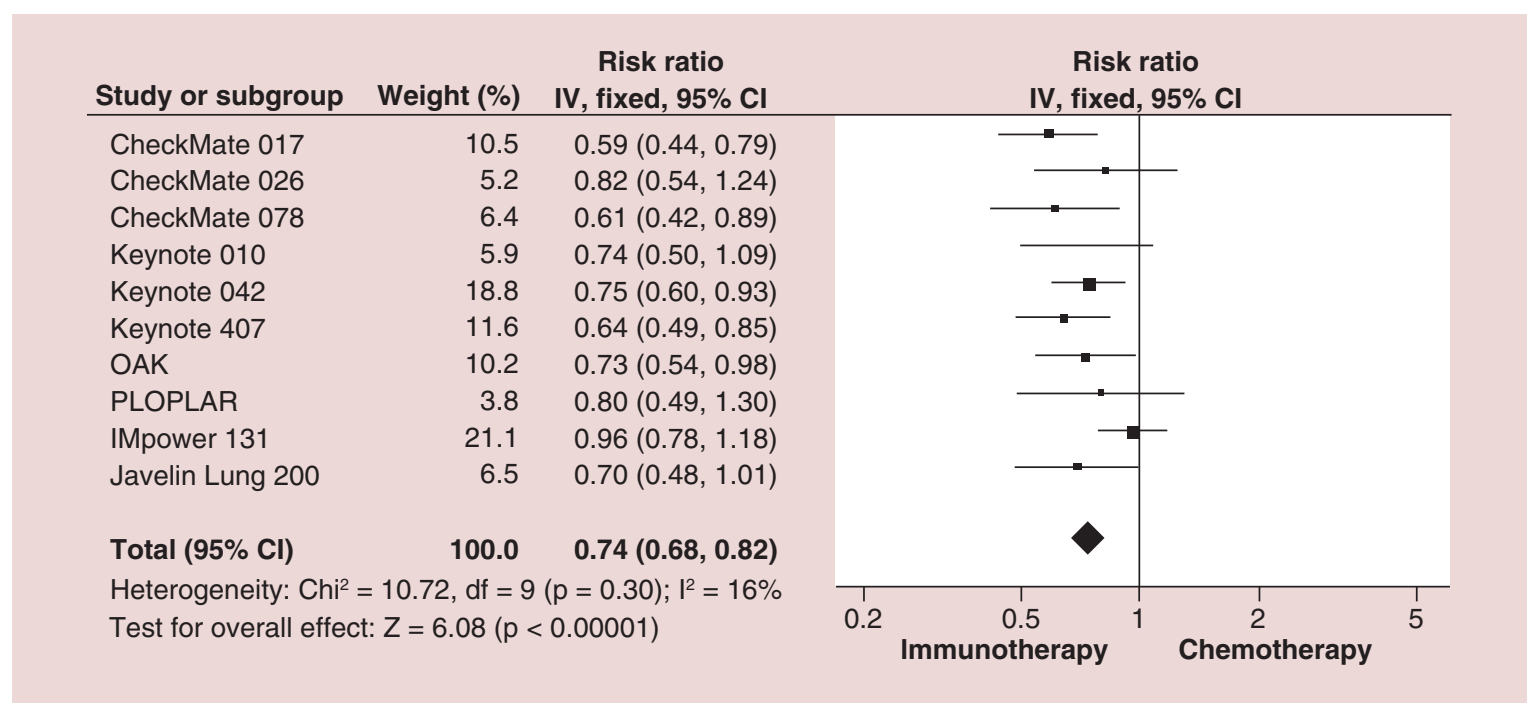

Figure 2. Forest plot for primary outcome analysis on overall survival. IV: Inverse variance.

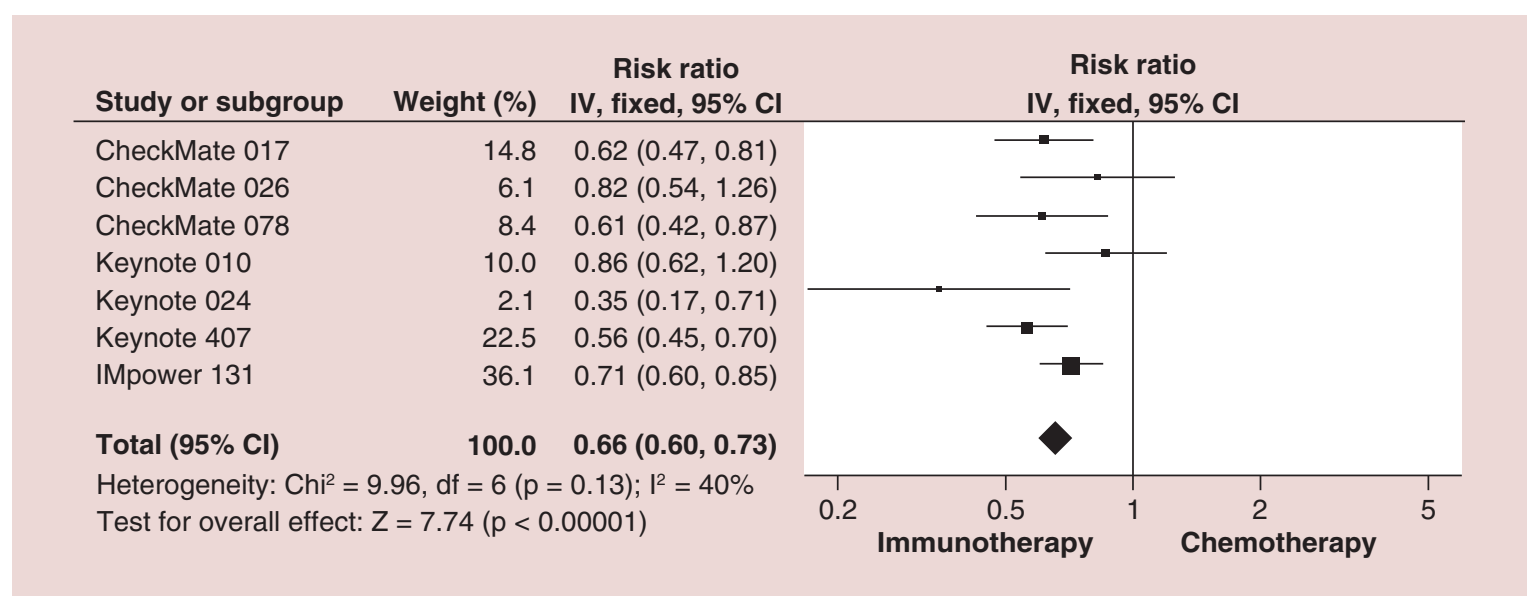

Figure 3. Forest plot for the analysis on progression-free survival. IV: Inverse variance.

Characteristics and the quality assessment of the included studies are presented in Table 1 . The eligible studies were published from 2015 to 2019 and the sample size of each study ranged from 56 to 683 . The median follow-up time ranged from 7.8 to 21.0 months.

\section{OS benefit from PD-1/PD-L1 inhibitors}

Squamous-cell lung cancer patients treated with PD-1/PD-L1 inhibitors received superior OS to chemotherapy. Pooled HRs based on ten studies demonstrated lower risks of death with PD-1/PD-L1 inhibitors when compared with chemotherapy (HR: 0.74; 95\% CI: 0.68-0.82; $\mathrm{p}<0.001$ ) (Figure 2). Low heterogeneity was observed for OS with no significant difference $\left(\mathrm{I}^{2}=16 \% ; \mathrm{p}=0.30\right)$.

\section{PFS benefit from PD-1/PD-L1 inhibitors}

A significant improvement in PFS was found among advanced squamous-cell NSCLC patients treated with PD1/PD-L1 inhibitors (HR: 0.66; 95\% CI: 0.60-0.73; $\mathrm{p}<0.001$ ) compared with those treated with chemotherapy (Figure 3). No significant heterogeneity was found for PFS $\left(\mathrm{I}^{2}=40 \% ; \mathrm{p}=0.13\right)$. 


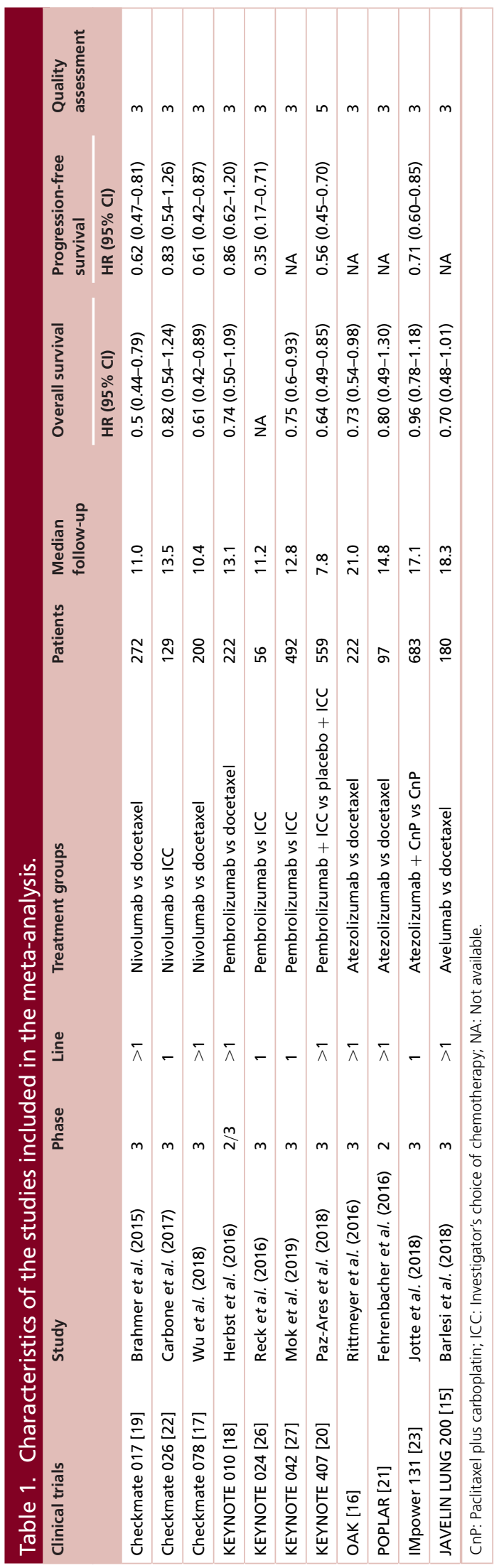




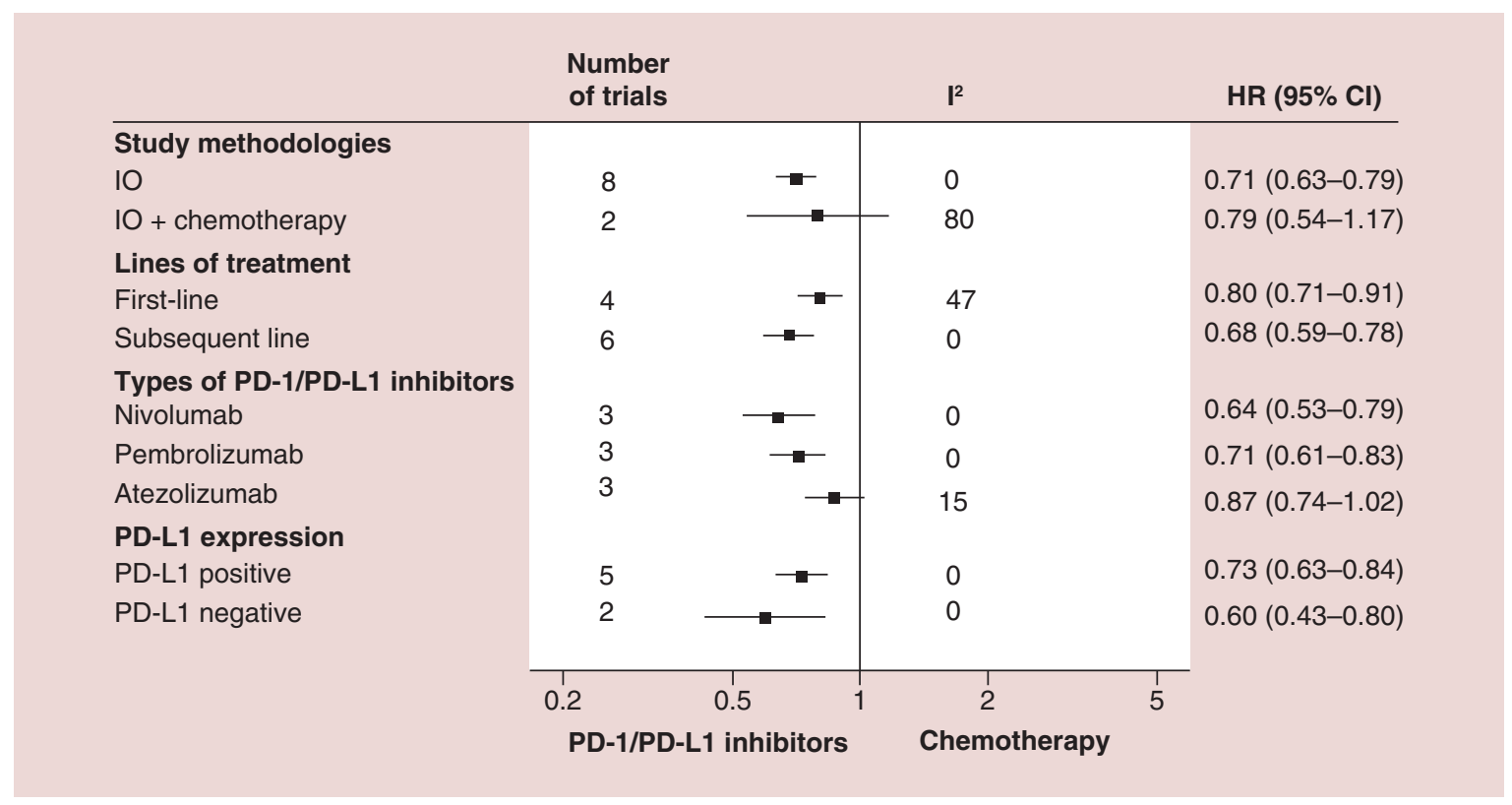

Figure 4. Subgroup analyses on overall survival according to study methodologies, lines of treatment, types of PD-1/PD-L1 inhibitors and PD-L1 expression.

HR: Hazard ratio; IO: Immunotherapy; IO+Chemotherapy: The combination of immunotherapy and chemotherapy; PD-1: Programmed death-1; PD-L1: Programmed death-ligand-1.

\section{Subgroup analysis}

We performed subgroup analyses according to lines of treatment (first line and subsequent lines), types of PD1/PD-L1 inhibitors (nivolumab, pembrolizumab and atezolizumab), study methodologies (immunotherapy vs chemotherapy, immunotherapy plus chemotherapy vs chemotherapy alone) and PD-L1 expression (Supplementary Table 1).

The OS benefit of PD-1/PD-L1 inhibitors for squamous NSCLC patients was similar in the first-line and subsequent line settings, PD-L1 expression and two different study methodologies. We found no OS advantage in advanced squamous NSCLC patients treated with atezolizumab (HR: 0.87; 95\% CI: 0.74-1.02; p = 0.087). In contrast, OS was significantly prolonged for those treated with nivolumab and pembrolizumab (nivolumab, HR: 0.64; 95\% CI: 0.53-0.79; p < 0.001; pembrolizumab, HR: 0.71; 95\% CI: 0.61-0.83; p < 0.001) (Figure 4). The PFS benefit of PD-1/PD-L1 inhibitors over chemotherapy was equally found in the presence or absence of PD-L1 expression, different study methodologies, lines of treatment and types of PD-1/PD-L1 inhibitors (Figure 5).

\section{Study quality \& publication bias}

Randomized treatment allocation sequences were generated in all trials. Ten trials were open label whereas one trial was double blinded. The Jadad score ranged from 3 to 5 with a mean score of 3.2, indicating that the quality was high (Table 1). No study received a low-quality score. The funnel plot, as well as Egger's and Begg's tests, demonstrated that no publication bias in overall or subgroup populations (all $\mathrm{p}>0.05$ ).

\section{Discussion}

Single agent PD-1/PD-L1 inhibitors and PD-1/PD-L1 inhibitors plus chemotherapy have been explored as treatment strategies for advanced NSCLC patients. However, for advanced squamous NSCLC patients, the available therapies were limited with poor efficacy. We conducted this meta-analysis to investigate the efficacy of PD-1/PD-L1 inhibitors versus chemotherapy for squamous NSCLC patients.

Our study included 11 clinical trials involving 3112 patients, which compared the efficacy of PD-1/PD-L1 inhibitors with chemotherapy for advanced squamous NSCLC patients. We observed that PD-1/PD-L1 inhibitors significantly improved OS and PFS of advanced squamous-cell lung cancer when compared with chemotherapy (OS, HR: 0.74; 95\% CI: 0.68-0.82; p < 0.001; PFS, HR: 0.66; 95\% CI: 0.60-0.73; p < 0.001). To the best of 


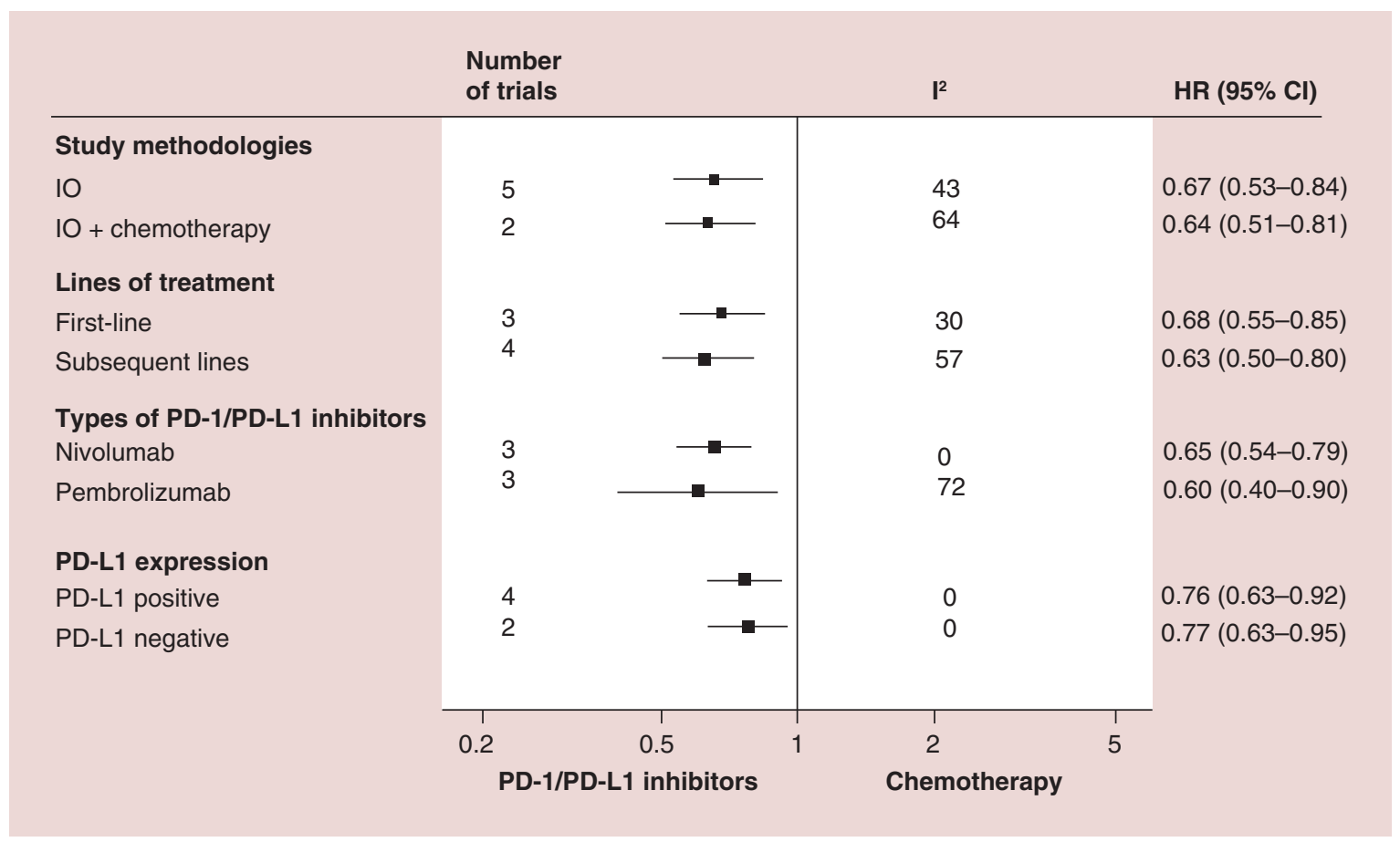

Figure 5. Subgroup analyses on progression-free survival according to study methodologies, lines of treatment, types of PD-1/PD-L1 inhibitors and PD-L1 expression.

HR: Hazard ratio; IO: Immunotherapy; IO+Chemotherapy: The combination of immunotherapy and chemotherapy; PD-1: Programmed death-1; PD-L1: Programmed death-ligand-1.

our knowledge, this is the first meta-analysis to explore the efficacy of PD-1/PD-L1 inhibitors on both OS and PFS for metastatic squamous NSCLC patients.

The results of our study are consistent with previous studies. The subgroup analyses in Huang et al. showed that advanced squamous NSCLC patients treated with PD-1/PD-L1 inhibitors had improved OS compared with those treated with chemotherapy (HR: 0.68; 95\% CI: 0.58-0.80), similar to our results. Squamous-cell lung cancer patients treated with nivolumab showed superior PFS and OS to chemotherapy (PFS, HR: 95\% CI; OS, HR: 0.59, 95\% CI: 0.44-0.79) in Checkmate 017 [19]. KEYNOTE 407 showed that OS was significantly prolonged for squamous NSCLC patients with pembrolizumab plus chemotherapy [20]. However, the Huang et al. study only included six clinical trials and focused only on the analysis of OS [28]. Our meta-analysis was performed on 11 trials, including the latest published studies, and it evaluated both PFS and OS of PD-1/PD-L1 inhibitors in advanced squamous-cell lung cancer patients. Squamous NSCLC patients were reported to carry a high mutation load with an average of 11.8 somatic mutations per $\mathrm{Mb}$ [29]. Tumor mutation load is considered a strong predictive biomarker for the efficacy of immunotherapy [30]. This may explain why patients with advanced squamous-cell lung cancer might benefit from improved on OS and PFS when treated with immunotherapy.

We have performed some subgroup analyses on OS and PFS, involving study methodologies, treatment lines, types of PD-1/PD-L1 inhibitors and PD-L1 expression. In all subgroups, PD-1/PD-L1 inhibitors significantly prolonged OS in immunotherapy, except the atezolizumab subgroup. Patients treated with atezolizumab showed no significant difference on OS between PD-1/PD-L1 inhibitors and chemotherapy. IMpower 131 study showed improved PFS in squamous NSCLC patients treated with atezolizumab plus chemotherapy, but no significant difference in OS [23]. A similar result also could be found for OS in patients with squamous disease from POPLAR trials, with no significant HR of 0.80 (95\% CI: 0.49-1.30) [21]. However, Checkmate 017 demonstrated that nivolumab significantly prolonged PFS and OS in squamous NSCLC patients [19]. Furthermore, pembrolizumab combined with chemotherapy also significantly improved PFS and OS compared with chemotherapy alone in KEYNOTE 407 [20]. A network meta-analysis reported that pembrolizumab resulted in significantly superior OS (HR: $0.67 ; \mathrm{p}=0.02$ ) and numerically better PFS (HR: 0.79; $\mathrm{p}=0.10)$ than atezolizumab in advanced squamouscell lung cancer patients [31]. This difference may be a result of varied abilities of PD-1 or PD-L1 receptors 
among different kinds of immune checkpoint inhibitors. In addition, the immune microenvironment of squamous NSCLC may be not easily simulated by atezolizumab. We assumed that atezolizumab might not be the first choice for squamous NSCLC patients treated with immunotherapy. Subgroup analysis of PD-L1 expression showed that squamous NSCLC patients obtained prolonged PFS and OS from immunotherapy, regardless of the expression of the PD-L1 protein, which was also observed in the results of Checkmate 017 [19]. Thus, immunotherapy could be an optional treatment strategy for advanced squamous NSCLC patients.

The major strength of our study was the high quality of the data included in the meta-analysis. All randomized trials and subgroups from several trials comparing immunotherapy with chemotherapy, involving 3112 squamous NSCLC patients from most up-to-date publications, were available for our study. Another strength was the low level of heterogeneity between studies.

We observed several limitations in the present study. First, one trial (IMpower 131) has not yet been published and is available as only an ASCO meeting abstract. Thus, the data for IMpower 131 were limited from the ASCO meeting abstract [23]. Second, our analysis depended on published results from data of patients in clinical trials rather than of each individual patient's data. Furthermore, some of squamous NSCLC patients in our study were from subgroup analyses. Therefore, we could not collect important information about the patients, such as gender, smoking history, PD-L1 expression and adverse events of immunotherapy.

\section{Conclusion}

In summary, treatment with PD-1/PD-L1 inhibitors resulted in significantly longer OS and PFS in advanced squamous NSCLC patients compared with chemotherapy. With improved PFS and OS, immunotherapy may be an optional treatment for squamous NSCLC patients.

\section{Future perspective}

Squamous-cell lung cancer is one of the most common histological subtypes for NSCLC. However, patients with squamous-cell lung cancer experienced finite advantage of chemotherapy and target drugs. Our findings highlight the superiority of PD-1/PD-L1 inhibitors for advanced squamous NSCLC patients versus chemotherapy. Our study showed that atezolizumab may provide limited advantage in OS for advanced squamous NSCLC patients, but this possibility requires more confirmatory evidence. Immune microenvironment of squamous cell carcinoma should be deeply investigated in the future. Besides, it is urgent to identify a predictive model with molecular profiling data such as tumor mutation burden, PD-L1 expression or infiltrating CD8 ${ }^{+}$cells for squamous NSCLC in this era of immunotherapy.

\section{Financial \& competing interests disclosure}

This study was supported by grant from the National Natural science Foundation of China (grant number 81573780) and the Natural science Foundation of Guangdong Provincial (grant number 2018B030311014). The authors have no other relevant affiliations or financial involvement with any organization or entity with a financial interest in or financial conflict with the subject matter or materials discussed in the manuscript apart from those disclosed.

No writing assistance was utilized in the production of this manuscript.

Ethical conduct of research

The authors stated that they have obtained appropriate institutional review board of approval or have followed the principles outlined in the Declaration of Helsinki for all human or animal experimental investigations.

Open access

This work is licensed under the Attribution-NonCommercial-NoDerivatives 4.0 Unported License. To view a copy of this license, visit http://creativecommons.org/licenses/by-nc-nd/4.0/ 
Summary points

\section{Background}

- Squamous-cell carcinoma accounts for approximately $20-30 \%$ of all cases of lung cancer.

- Limited treatment strategies are available for patients with advanced squamous-cell lung cancer.

- The development of immune checkpoint inhibitors has become a paradigm shift in the therapeutic strategies for non-small-cell lung cancer (NSCLC) patients.

- It is necessary to perform a meta-analysis to explore the efficacy of checkpoint inhibitors for advanced squamous-cell lung cancer patients.

\section{Materials \& methods}

- We utilized clinical trials published in Pubmed, Embase and the Cochrane library. Abstracts published from main international meetings were also considered.

- We performed this meta-analysis to compare the benefits of immunotherapy and chemotherapy for advanced squamous-cell lung cancer patients. We adopted a random or fixed effect model based on the heterogeneity between trials.

Results

- A total of 11 randomized controlled trials involving 3112 patients with advanced squamous-cell NSCLC were included.

- Compared with chemotherapy, PD-1/PD-L1 inhibitors significantly improved overall survival (OS) (hazard ratio: 0.74; $p<0.001$ ) and profession-free survival (PFS) (hazard ratio: $0.66 ; p<0.001$ ) in metastatic squamous-cell NSCLC patients.

- The OS and PFS benefits of treatment with PD-1/PD-L1 inhibitors were similar in the subgroup analyses of line settings, PD-L1 expression and different study methodologies.

- There was no OS advantage in advanced squamous NSCLC patients with atezolizumab.

Conclusion

- Treatment with PD-1/PD-L1 inhibitors improved OS and PFS in advanced squamous NSCLC patients, compared with chemotherapy.

\section{References}

1. Siegel RL, Miller KD, Jemal A. Cancer statistics, 2019. CA Cancer J. Clin. 69(1), 7-34 (2019).

2. Travis WD. Pathology of lung cancer. Clin. Chest Med. 32(4), 669-692 (2011).

3. Solomon BJ, Mok T, Kim DW et al. First-line crizotinib versus chemotherapy in ALK-positive lung cancer. N. Engl. J. Med. 371(23), 2167-2177 (2014).

4. Maemondo M, Inoue A, Kobayashi K et al. Gefitinib or chemotherapy for non-small-cell lung cancer with mutated EGFR. N. Engl. J. Med. 362(25), 2380-2388 (2010).

5. An SJ, Chen ZH, Su J et al. Identification of enriched driver gene alterations in subgroups of non-small-cell lung cancer patients based on histology and smoking status. PLoS ONE 7(6), e40109 (2012).

6. Scagliotti G, Hanna N, Fossella F et al. The differential efficacy of pemetrexed according to NSCLC histology: a review of two Phase III studies. Oncologist 14(3), 253-263 (2009).

7. Sandler A, Gray R, Perry MC et al. Paclitaxel-carboplatin alone or with bevacizumab for non-small-cell lung cancer. N. Engl. J. Med. 355(24), 2542-2550 (2006).

8. Thatcher N, Hirsch FR, Luft AV et al. Necitumumab plus gemcitabine and cisplatin versus gemcitabine and cisplatin alone as first-line therapy in patients with stage IV squamous non-small-cell lung cancer (SQUIRE): an open-label, randomised, controlled Phase III trial. Lancet Oncol. 16(7), 763-774 (2015).

9. Socinski MA, Bondarenko I, Karaseva NA et al. Weekly nab-paclitaxel in combination with carboplatin versus solvent-based paclitaxel plus carboplatin as first-line therapy in patients with advanced non-small-cell lung cancer: final results of a Phase III trial. J. Clin. Oncol. 30(17), 2055-2062 (2012).

10. Fusi A, Festino L, Botti G et al. PD-L1 expression as a potential predictive biomarker. Lancet Oncol. 16(13), 1285-1287 (2015).

11. Pardoll DM. The blockade of immune checkpoints in cancer immunotherapy. Nat. Rev. Cancer 12(4), 252-264 (2012).

12. Borghaei H, Paz-Ares L, Horn L et al. Nivolumab versus docetaxel in advanced nonsquamous non-small-cell lung cancer. N. Engl. J. Med. 373(17), 1627-1639 (2015).

13. Ferris RL, Blumenschein G Jr, Fayette J et al. Nivolumab for recurrent squamous-cell carcinoma of the head and neck. N. Engl. J. Med. 375(19), 1856-1867 (2016).

14. Larkin J, Minor D, D'angelo S et al. Overall survival in patients with advanced melanoma who received nivolumab versus investigator's choice chemotherapy in CheckMate 037: a randomized, controlled, open-label Phase III trial. J. Clin. Oncol. 36(4), 383-390 (2018). 
15. Barlesi F, Vansteenkiste J, Spigel D et al. Avelumab versus docetaxel in patients with platinum-treated advanced non-small-cell lung cancer (JAVELIN Lung 200): an open-label, randomised, Phase III study. Lancet Oncol. 19(11), 1468-1479 (2018).

16. Rittmeyer A, Barlesi F, Waterkamp D et al. Atezolizumab versus docetaxel in patients with previously treated non-small-cell lung cancer (OAK): a Phase III, open-label, multicentre randomised controlled trial. Lancet 389(10066), 255-265 (2017).

17. Wu YL, Lu S, Cheng Y et al. Nivolumab versus docetaxel in a predominantly Chinese patient population with previously treated advanced NSCLC: CheckMate 078 randomized Phase III clinical trial. J. Thorac. Oncol. 14(5), 867-875 (2019).

18. Herbst RS, Baas P, Kim DW et al. Pembrolizumab versus docetaxel for previously treated, PD-L1-positive, advanced non-small-cell lung cancer (KEYNOTE-010): a randomised controlled trial. Lancet 387(10027), 1540-1550 (2016).

19. Brahmer J, Reckamp KL, Baas P et al. Nivolumab versus docetaxel in advanced squamous-cell non-small-cell lung cancer. N. Engl. J. Med. 373(2), 123-135 (2015).

20. Paz-Ares L, Luft A, Vicente D et al. Pembrolizumab plus chemotherapy for squamous non-small-cell lung cancer. N. Engl. J. Med. 379(21), 2040-2051 (2018).

21. Fehrenbacher L, Spira A, Ballinger M et al. Atezolizumab versus docetaxel for patients with previously treated non-small-cell lung cancer (POPLAR): a multicentre, open-label, Phase II randomised controlled trial. Lancet 387(10030), 1837-1846 (2016).

22. Carbone DP, Reck M, Paz-Ares L et al. First-line nivolumab in stage IV or recurrent non-small-cell lung cancer. N. Engl. J. Med. 376(25), 2415-2426 (2017).

23. Jotte Rm, Cappuzzo F, Vynnychenko I et al. IMpower131: primary PFS and safety analysis of a randomized Phase III study of atezolizumab+carboplatin+paclitaxel or nab-paclitaxel vs carboplatin+nab-paclitaxel as $1 \mathrm{~L}$ therapy in advanced squamous NSCLC. $J$. Clin. Oncol. 36(Suppl. 18), LBA9000 (2018).

24. Jadad AR, Moore RA, Carroll D et al. Assessing the quality of reports of randomized clinical trials: is blinding necessary? Control. Clin. Trials 17(1), 1-12 (1996).

25. Higgins JP, Thompson SG, Deeks JJ, Altman DG. Measuring inconsistency in meta-analyses. BMJ 327(7414), 557-560 (2003)

26. Reck M, Rodriguez-Abreu D, Robinson AG et al. Pembrolizumab versus chemotherapy for PD-L1-positive non-small-cell lung cancer. N. Engl. J. Med. 375(19), 1823-1833 (2016).

27. Mok TSK, Wu YL, Kudaba I et al. Pembrolizumab versus chemotherapy for previously untreated, PD-L1-expressing, locally advanced or metastatic non-small-cell lung cancer (KEYNOTE-042): a randomised, open-label, controlled, Phase III trial. Lancet 393(10183), 1819-1830 (2019).

28. Huang Q, Zhang H, Hai J et al. Impact of PD-L1 expression, driver mutations and clinical characteristics on survival after anti-PD-1/PD-L1 immunotherapy versus chemotherapy in non-small-cell lung cancer: a meta-analysis of randomized trials. Oncoimmunology 7(12), e1396403 (2018).

29. Zhang XC, Wang J, Shao GG et al. Comprehensive genomic and immunological characterization of Chinese non-small-cell lung cancer patients. Nat. Commun. 10(1), 1772 (2019).

30. Yarchoan M, Hopkins A, Jaffee EM. Tumor mutational burden and response rate to PD-1 inhibition. N. Engl. J. Med. 377(25), 2500-2501 (2017).

31. Zhang $\mathrm{Y}$, Zhou $\mathrm{H}$, Zhang $\mathrm{L}$. Which is the optimal immunotherapy for advanced squamous non-small-cell lung cancer in combination with chemotherapy: anti-PD-1 or anti-PD-L1? J. Immunother. Cancer 6(1), 135 (2018). 